\title{
Identification of signaling pathways, matrix-digestion enzymes, and motility components controlling Vibrio cholerae biofilm dispersal
}

\author{
Andrew A. Bridges ${ }^{a, b}\left(\mathbb{D}\right.$, Chenyi Fei ${ }^{a}{ }^{(0)}$, and Bonnie L. Bassler ${ }^{a, b, 1}$

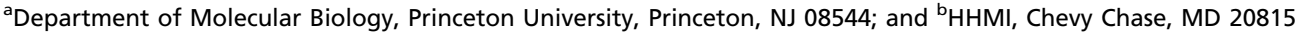 \\ Contributed by Bonnie L. Bassler, October 30, 2020 (sent for review October 9, 2020; reviewed by Susan Gottesman and Jun Zhu)
}

Bacteria alternate between being free-swimming and existing as members of sessile multicellular communities called biofilms. The biofilm lifecycle occurs in three stages: cell attachment, biofilm maturation, and biofilm dispersal. Vibrio cholerae biofilms are hyperinfectious, and biofilm formation and dispersal are considered central to disease transmission. While biofilm formation is well studied, almost nothing is known about biofilm dispersal. Here, we conducted an imaging screen for $\boldsymbol{V}$. cholerae mutants that fail to disperse, revealing three classes of dispersal components: signal transduction proteins, matrix-degradation enzymes, and motility factors. Signaling proteins dominated the screen and among them, we focused on an uncharacterized two-component sensory system that we term DbfS/DbfR for dispersal of biofilm sensor/regulator. Phospho-DbfR represses biofilm dispersal. DbfS dephosphorylates and thereby inactivates $\mathrm{DbfR}$, which permits dispersal. Matrix degradation requires two enzymes: LapG, which cleaves adhesins, and $\mathrm{RbmB}$, which digests matrix polysaccharides. Reorientation in swimming direction, mediated by CheY3, is necessary for cells to escape from the porous biofilm matrix. We suggest that these components act sequentially: signaling launches dispersal by terminating matrix production and triggering matrix digestion, and subsequent cell motility permits escape from biofilms. This study lays the groundwork for interventions aimed at modulating $V$. cholerae biofilm dispersal to ameliorate disease.

biofilm dispersal | Vibrio cholerae | signal transduction | virulence | highcontent imaging

B acteria transition between existing in the biofilm state, in which cells are members of surface-associated multicellular collectives, and living as free-swimming, exploratory individuals. Biofilms consist of cells surrounded by a self-secreted extracellular matrix that protects the resident cells from threats including predation, antimicrobials, and dislocation due to flow (1-3). Biofilms are relevant to human health because beneficial microbiome bacteria exist in biofilms, and, during disease, because pathogens in biofilms evade host immune defenses, thwart medical intervention, and exhibit virulence (4-7). The biofilm lifecycle consists of three stages: cell attachment, biofilm maturation, and dispersal (Fig. 1A) (8). Cells liberated during the dispersal step can disseminate and found new biofilms (8). The environmental stimuli and the components facilitating biofilm attachment and maturation have been defined for many bacterial species (9). In contrast, little is known about the biofilm dispersal stage.

The model pathogen Vibrio cholerae forms biofilms in its aquatic habitat. Biofilm cells are especially virulent in mouse models of cholera disease, and biofilms are thought to be critical for cholera transmission (10-14). Studies of $V$. cholerae biofilms have focused predominantly on matrix-overproducing strains that constitutively exist in the biofilm mode and that do not disperse. This research approach has propelled understanding of $V$. cholerae biofilm attachment and maturation, revealing that the second messenger cyclic diguanylate (c-di-GMP) is a master regulator of biofilm formation, and that expression of vibrio polysaccharide (vps) biosynthetic genes are required (15-17). The strategy of characterizing constitutive biofilm formers, while successful for uncovering factors that promote biofilm formation, has necessarily precluded studies of biofilm dispersal. Here, we used a microscopy assay that allowed us to monitor the full wildtype (WT) $V$. cholerae biofilm lifecycle. We combined this assay with high-content imaging of randomly mutagenized WT $V$. cholerae to identify genes required for biofilm dispersal. Investigation of the proteins encoded by the genes allowed us to characterize the signaling relays, matrix-digestion enzymes, and motility components required for biofilm dispersal, a key stage in the lifecycle of the global pathogen $V$. cholerae.

\section{Results}

We previously developed a brightfield microscopy assay that allows us to monitor the full WT $V$. cholerae biofilm lifecycle in real time (18). In our approach, $V$. cholerae cells are inoculated onto glass coverslips at low cell density, and brightfield timelapse microscopy is used to monitor biofilm progression. WT biofilms reach peak biomass after 8 to $9 \mathrm{~h}$ of incubation, and subsequently, dispersal occurs and is completed by 12 to $13 \mathrm{~h}$ (Fig. $1 B$ and $C$ ). To identify genes required for biofilm dispersal,

\section{Significance}

The global pathogen Vibrio cholerae alternates between free swimming and existing in sessile multicellular communities known as biofilms. Transitioning between these lifestyles is key for disease transmission. $V$. cholerae biofilm formation is well studied; however, almost nothing is known about how $V$. cholerae cells disperse from biofilms, precluding our understanding of a central pathogenicity step. Here, we conducted an imaging screen for $\boldsymbol{V}$. cholerae mutants that failed to disperse. Our screen revealed three classes of components required for dispersal: signal transduction, matrix degradation, and motility factors. We characterized these components to reveal the sequence of molecular events that choreograph $V$. cholerae biofilm dispersal. Our report provides a framework for developing strategies to modulate biofilm dispersal to prevent or treat disease.

Author contributions: A.A.B. and B.L.B. designed research; A.A.B. and C.F. performed research; A.A.B. and C.F. contributed new reagents/analytic tools; A.A.B., C.F., and B.L.B. analyzed data; and A.A.B., C.F., and B.L.B. wrote the paper.

Reviewers: S.G., National Institutes of Health; and J.Z., University of Pennsylvania School of Medicine.

Competing interest statement: B.L.B. and S.G. are co-authors on a 2017 comment article concerning gender discrimination in science. The piece has a total of 37 co-authors.

This open access article is distributed under Creative Commons Attribution License 4.0 (CC BY).

${ }^{1}$ To whom correspondence may be addressed. Email: bbassler@princeton.edu.

This article contains supporting information online at https://www.pnas.org/lookup/suppl/ doi:10.1073/pnas.2021166117/-/DCSupplemental.

First published December 7, 2020. 

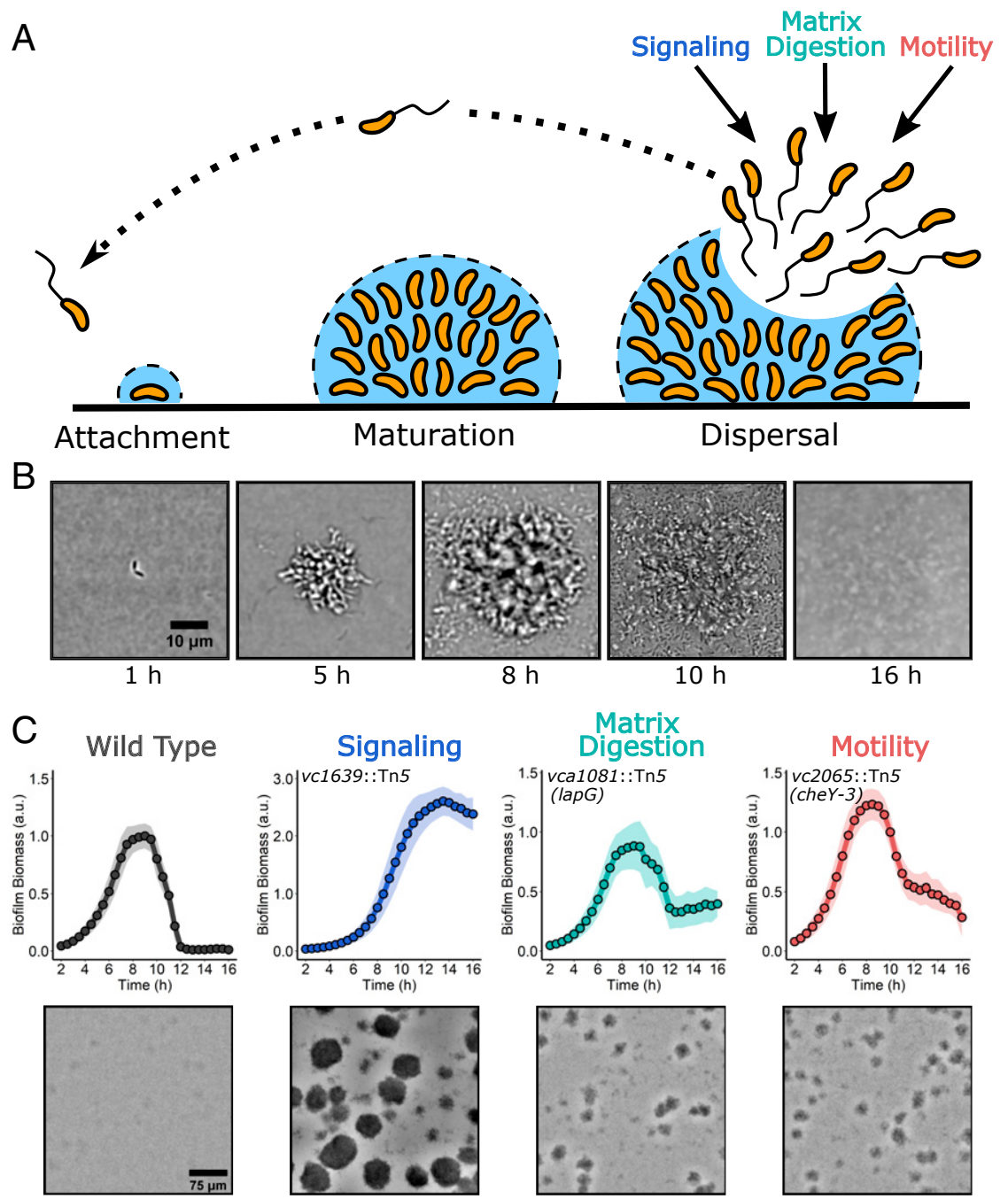

Fig. 1. A high-content imaging screen identified genes required for $V$. cholerae biofilm dispersal. (A) Schematic illustrating the $V$. cholerae biofilm lifecycle. See text for details. (B) Brightfield image series over time of the WT $V$. cholerae biofilm lifecycle. $(C, T o p)$ Quantitation of biofilm biomass over time as measured by time-lapse microscopy for WT and representative transposon insertion mutants from each of the three functional categories identified in the screen. Note the differences in $y$-axis scales. Data are represented as means normalized to the peak biofilm biomass of the WT strain. $n=3$ biological replicates and $n=3$ technical replicates, \pm SD (shaded). a.u., arbitrary unit. (C, Bottom) Representative brightfield images of biofilms at the final $16 \mathrm{~h}$ timepoint for the strains presented in C, Top.

we combined mutagenesis with high-content imaging of the output of this assay. Specifically, WT $V$. cholerae was mutagenized with Tn5, yielding approximately 7,000 mutants that were arrayed in 96-well plates. Following overnight growth, the mutants were diluted to low cell density in minimal medium, a condition that drives initiation of the biofilm lifecycle. Brightfield images of each well were captured at $8 \mathrm{~h}$ postinoculation to assess biofilm maturation and at $13 \mathrm{~h}$ to evaluate biofilm dispersal. Mutants that showed no defects in biofilm maturation on the $8 \mathrm{~h}$ images but displayed significant remaining biofilm biomass on the $13 \mathrm{~h}$ images were identified.

To verify phenotypes, candidate mutants were individually reevaluated by time-lapse microscopy. Mutants that accumulated at the bottom of wells due to aggregation or that failed to attach to surfaces were excluded from further analysis, eliminating strains harboring insertions in $\mathrm{O}$-antigen and flagellar genes, respectively. The locations of transposon insertions in the 47 mutants that met our criteria were defined and corresponded to 10 loci. The new genes from the screen fell into three classes: signal transduction (blue), matrix degradation (green), and motility (red) (Fig. $1 A$ and $C$ ). In-frame deletions of each gene were constructed, and the biofilm lifecycles of the deletion mutants were imaged to confirm that the genes were required for biofilm dispersal (Table 1 and Movie S1). We also identified insertions in genes encoding proteins with known roles in biofilm dispersal (i.e., RpoS and quorum sensing), which we excluded from further analysis $(18,19)$.

Proteins involved in signal transduction dominated the screen (7 of 10 loci) and included the ribosome-associated GTPase, BipA, multiple c-di-GMP signaling proteins, polyamine signaling proteins, and a putative two-component histidine kinase, Vc1639. The signal transduction mutants displayed biofilm dispersal phenotypes of varying severity. The $\Delta b i p A$ strain showed a modest defect, with $\sim 19 \%$ of its biofilm biomass remaining at $16 \mathrm{~h}$, the final timepoint of our data acquisition, while the WT had $\sim 6 \%$ of its biomass remaining at this timepoint. In contrast, the $\Delta v c 1639$ mutant underwent no appreciable dispersal (Table 1). In the category of matrix degradation, two enzymes were identified: LapG, a periplasmic protease, and $\mathrm{RbmB}$, a putative polysaccharide lyase (Table 1). A single motility mutant was identified with an insertion in the gene encoding the chemotaxis response regulator, cheY3 (Table 1). Below, we report mechanistic studies 
Table 1. Genes identified as required for $\boldsymbol{V}$. cholerae biofilm dispersal and phenotypes of deletion mutants

\begin{tabular}{|c|c|c|c|c|c|c|}
\hline Gene & Function & $\begin{array}{l}\text { Functional } \\
\text { category }\end{array}$ & $\begin{array}{c}\text { Times } \\
\text { hit }\end{array}$ & $\begin{array}{c}\text { Peak biomass (vs. WT) } \\
\text { mean } \pm S D\end{array}$ & $\begin{array}{l}\text { Peak time, } h, \\
\text { mean } \pm S D\end{array}$ & $\begin{array}{l}\text { Biomass remaining at } 16 \mathrm{~h}, \%, \\
\text { mean } \pm \mathrm{SD}\end{array}$ \\
\hline WT & - & - & - & $1.0 \pm 0.2$ & $8.7 \pm 0.4$ & $6 \pm 4$ \\
\hline bipA (vc2744) & Ribosome-associated GTPase & Signaling & 2 & $1.0 \pm 0.2$ & $9.6 \pm 0.3$ & $19 \pm 6$ \\
\hline cdgl (vc0658) & c-di-GMP phosphodiesterase & Signaling & 1 & $0.9 \pm 0.2$ & $8.7 \pm 0.4$ & $17 \pm 9$ \\
\hline $\operatorname{rocs}(v c 0653)$ & c-di-GMP phosphodiesterase & Signaling & 1 & $1.3 \pm 0.3$ & $10.4 \pm 0.6$ & $59 \pm 13$ \\
\hline potD1 (vc1424) & Polyamine transporter & Signaling & $6^{*}$ & $1.6 \pm 0.2$ & $11.9 \pm 0.9$ & $90 \pm 12$ \\
\hline$d b f S(v c 1639)$ & Histidine kinase & Signaling & 8 & $1.8 \pm 0.3$ & $14.3 \pm 0.9$ & $95 \pm 8$ \\
\hline lapG (vca1081) & Peptidase & $\begin{array}{c}\text { Matrix } \\
\text { digestion }\end{array}$ & 3 & $0.8 \pm 0.2$ & $9.4 \pm 0.2$ & $55 \pm 12$ \\
\hline$r b m B(v c 0929)$ & Polysaccharide lyase & $\begin{array}{c}\text { Matrix } \\
\text { digestion }\end{array}$ & 21 & $0.9 \pm 0.2$ & $10 \pm 0.4$ & $69 \pm 12$ \\
\hline
\end{tabular}

*Value includes transposon insertions in other genes in this operon.

of select mutants from each category to define the functions of the components; other mutants will be characterized in separate reports.

\section{A Two-Component Regulatory System Controls V. cholerae Biofilm Dispersal. The mutant from our screen that exhibited the most extreme dispersal phenotype had a transposon in a gene encoding an uncharacterized putative histidine kinase (desig- nated HK), Vc1639 (Table 1). A screen for factors required for $V$. cholerae colonization of the suckling mouse intestine repeat- edly identified Vc1639, suggesting that this HK is core to the cholera disease (20). HKs typically contain periplasmic ligand- binding domains and internal catalytic domains that switch be- tween kinase and phosphatase activities based on ligand detection (21). HKs transmit sensory information to cognate response reg- ulators (RRs) by altering RR phosphorylation (22), and RRs in turn control gene expression and/or behavior depending on their phosphorylation states. Deletion of $v c 1639$ in $V$. cholerae resulted in an $80 \%$ increase in peak biofilm biomass relative to WT, and nearly all of the biofilm biomass remained at $16 \mathrm{~h}$, demonstrating that Vc1639 is essential for biofilm dispersal (Fig. $2 A$ and Table 1 ).}

Complementation of the $\Delta v c 1639$ mutant with $v c 1639$ inserted onto the chromosome at an ectopic locus restored WT biofilm dispersal (SI Appendix, Fig. S1A). Consistent with the extreme dispersal phenotype of the $\Delta v c 1639$ mutant, vpsL-lux expression was elevated 10 -fold throughout the growth curve in the $\Delta v c 1639$ strain compared with WT $V$. cholerae (Fig. $2 B$ ). vps $L$ is the first gene in the major extracellular matrix biosynthetic operon, showing that Vc1639 signaling regulates matrix production. We note that while expression was higher in the $\Delta v c 1639$ mutant than in the WT strain, expression decreased in both strains with increasing cell density (Fig. $2 B$ ). Quorum sensing is known to repress genes encoding matrix production components (23), likely underlying this effect. Fusions of lux promoter to the genes encoding the biofilm master regulators vpsR and vps $T$ also exhibited increased light production in the $\Delta v c 1639$ mutant, suggesting that Vc1639 acts at the top of the cascade to control global biofilm gene expression (SI Appendix, Fig. S1 $B$ and $C$ ).

$v c 1639$ is the final gene in a three-gene operon that includes genes encoding a hypothetical protein of unknown function (Vc1637) and an OmpR family RR (Vc1638) (Fig. 2C). We denote Vc1639 as DbfS, for dispersal of biofilm sensor, and Vc1638 as DbfR, for dispersal of biofilm regulator. Domain prediction suggests that DbfS contains two transmembrane (TM) domains, a periplasmic sensory domain, and a cytoplasmic HAMP domain that likely transmits ligand-binding-induced conformational changes to regulation of C-terminal kinase/ phosphatase activity (Fig. $2 C$ ). The first gene in the operon, $v c 1637$, appears to encode a small secreted protein containing a PepSY domain, a domain that inhibits extracellular proteases in other proteins (24). We did not examine the function of Vc1637 in this study.

To explore the connection between DbfS and DbfR in the control of biofilm dispersal, we deleted $d b f R$. Commonly, cognate HK and RR null mutants have identical phenotypes. To our surprise, the $\Delta d b f R$ mutant had no biofilm dispersal defect and progressed through the biofilm lifecycle identically to WT (Fig. 2D). We considered the possibility that some other RR is the partner to DbfS. To test this idea, we constructed the $\Delta d b f S$ $\triangle d b f R$ double mutant and found that this strain behaved identically to the $\Delta d b f R$ strain (Fig. $2 D$ ), demonstrating that $d b f R$ is epistatic to $d b f S$, and thus DbfR indeed functions downstream of DbfS. Moreover, because RRs are typically active when phosphorylated, our results suggest that DbfR must be active in the absence of DbfS. Thus, we reason that phospho-DbfR is the species present in the $\Delta d b f S$ strain.

To verify the hypothesis that phospho-DbfR is responsible for the dispersal defect in the $\Delta d b f S$ strain, we constructed a nonphosphorylatable allele of DbfR (D51V). The $V$. cholerae $d b f R^{D 51 V}$ mutant displayed the WT biofilm dispersal phenotype in the presence and the absence of DbfS (Fig. 2E). DbfR-SNAP fusions showed that SNAP did not interfere with WT DbfR function, and that DbfR protein abundance was unchanged in the $d b f R^{D 51 V}$ strain relative to WT (SI Appendix, Fig. S1 D and $E)$. Thus, phospho-DbfR causes $V$. cholerae cells to remain in the biofilm state in the $\Delta d b f S$ mutant.

It follows that deletion of $d b f S$ causes biofilm dispersal failure due to loss of DbfS phosphatase activity on DbfR. To test this hypothesis, we assessed in vivo DbfR phosphorylation in the presence and absence of DbfS. Phos-tag gel analysis enabled separation and visualization of phosphorylated and dephosphorylated DbfR. In the absence of DbfS, DbfR was phosphorylated, and induction of DbfS production caused the phospho-DbfR species to disappear (Fig. $2 F$ ). Thus, under our experimental conditions, DbfS functions as a DbfR phosphatase. We infer that some other unknown kinase must exist and it phosphorylates DbfR (Fig. $2 G$ ). We propose that phospho-DbfR is active and drives the expression of matrix biosynthetic genes, and that increased matrix production prevents biofilm dispersal. 

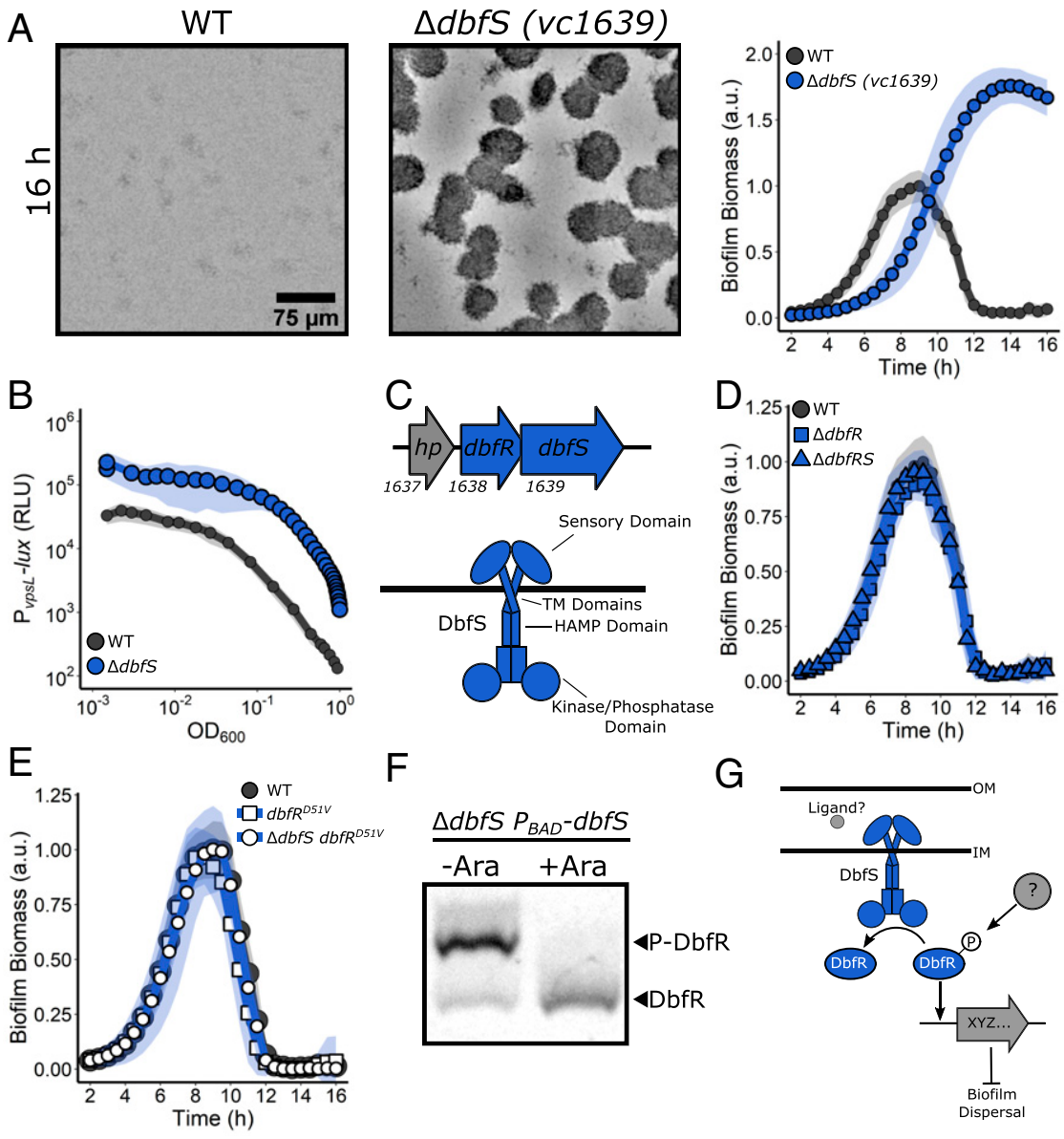

$\mathrm{F}$

G
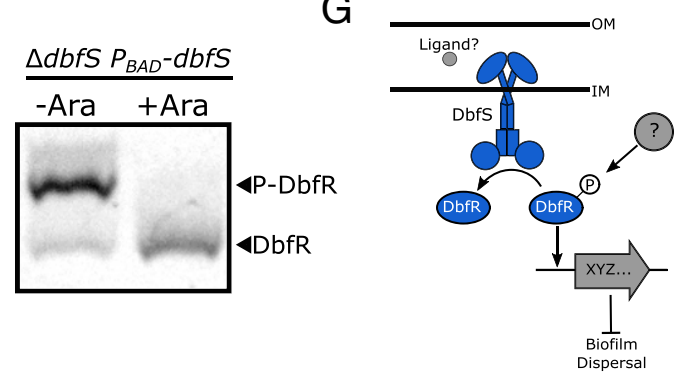

Fig. 2. A two-component system composed of DbfS (HK) and DbfR (RR) controls $V$. cholerae biofilm dispersal. ( $A$ ) Representative images at $16 \mathrm{~h}$ and quantitation of biofilm biomass over time measured by time-lapse microscopy for WT $V$. cholerae and the $\Delta d b f S$ (i.e., $\Delta v c 1639$ ) mutant. (B) The corresponding $P_{v p s}$-lux output for strains and growth conditions in $A$ over the growth curve. (C, Top) Operon structure of the genes encoding the DbfS-DbfR two-component system. (C, Bottom) Cartoon of the domain organization of DbfS. (D) As in $A$ for the $\Delta d b f R$ (i.e., $\Delta v c 1638$ ) strain and for the $\Delta d b f S \Delta d b f R$ double mutant. ( $E$ ) As in $A$ for the $d b f R^{D 51 V}$ and $\Delta d b f S d b f R^{D 51 V}$ strains. $(F)$ Representative Phos-tag gel analysis of DbfR-SNAP in the absence (-Ara) or presence (+Ara) of DbfS. Fucose was added to repress DbfS production in the uninduced samples. A phosphorylated protein migrates slower than the same unphosphorylated protein. (G) Proposed model for the DbfS-DbfR phosphorylation cascade regulating biofilm dispersal. OM, outer membrane; IM, inner membrane. In all biofilm measurements, $n=3$ biological replicates and $n=3$ technical replicates, \pm SD (shaded). For vpsL-lux measurements, $n=3$ biological replicates, \pm SD (shaded). The Phos-tag gel result is representative of $n=3$ independent biological replicates.

It is possible that phospho-DbfR also controls other genes involved in suppressing biofilm dispersal.

DbfS is well conserved in the vibrio genus; for example, in Vibrio vulnificus and Vibrio parahaemolyticus, DbfS has, respectively, $64 \%$ and $60 \%$ amino acid sequence identity to $V$. cholerae DbfS. In genera closely related to vibrio, such as allivibrio and photobacteria, the $d b f S$ gene exists in an identical operon organization, and the encoded protein shows high amino acid sequence identity ( $\sim 55$ to $65 \%$ ) to $V$. cholerae DbfS. In many of these cases, $d b f S$ is annotated as $p h o Q$, encoding the well-studied cation-regulated HK from enteric pathogens, including Escherichia coli and Salmonella. However, BLAST analysis of the DbfS protein sequence against that from $E$. coli $\mathrm{K}-12$ revealed limited homology to PhoQ, with $32 \%$ amino acid sequence identity (E value $=1 \mathrm{e}^{-41}$ ) and with the region of lowest similarity in the predicted ligand-binding domain. We tested whether the ligands that control PhoQ signal transduction also regulate DbfS-DbfR signaling (SI Appendix, Text and Fig. S2 $A-D$ ) and found that they do not. Thus, DbfS and DbfR are not functionally equivalent to PhoQ and its cognate RR, PhoP, respectively, and DbfS responds to an as-yet undefined stimulus to regulate biofilm dispersal.
Matrix Disassembly Mediates $\boldsymbol{V}$. cholerae Exit from Biofilms. The second group of mutants in our screen harbored insertions in the gene encoding the calcium-dependent periplasmic protease LapG that degrades outer-membrane-spanning adhesive proteins and in the gene specifying the extracellular polysaccharide lyase $\mathrm{RbmB}$ that degrades the VPS component of the biofilm matrix (25-27). The $\Delta l a p G$ strain exhibited slightly lower peak biofilm biomass compared to WT, with a short delay in the onset of dispersal, and $\sim 55 \%$ of its biomass remained at $16 \mathrm{~h}$ (Fig. $3 \mathrm{~A}$ and Table 1). The $\Delta l a p G$ and the WT strains had similar vpsLlux expression patterns (Fig. $3 B$ ), consistent with LapG playing no role in repression of matrix production but rather functioning downstream in matrix degradation. The LapG mechanism is known: when c-di-GMP concentrations are high, the FrhA and CraA adhesins are localized to the outer membrane, where they facilitate attachments important for biofilm formation (Fig. 3C) $(28,29)$. Under these conditions, LapG is sequestered and inactivated by the inner membrane c-di-GMP-sensing protein LapD (28). When c-di-GMP levels fall, LapD releases LapG, and LapG cleaves FrhA and CraA, facilitating cell detachment from biofilms (28). Our results are consistent with this 
mechanism; in the absence of LapG, FrhA and CraA remain intact, and $V$. cholerae cells cannot properly exit the biofilm state.

To verify that the established c-di-GMP-dependent regulatory mechanism controls LapG activity in our assay, we deleted lapD (Fig. $3 C$ ). Indeed, in the $\Delta l a p D$ strain, biofilm dispersal occurred prematurely, indicating that without LapD, LapG is not sequestered, and unchecked LapG activity promotes premature adhesin degradation and, consequently, early biofilm disassembly (Fig. $3 D$ ). The $\Delta l a p D \Delta l a p G$ double mutant had the same dispersal phenotype as the $\Delta l a p G$ single mutant, confirming that LapG functions downstream of LapD (Fig. 3D). Finally, in a reciprocal arrangement, overexpression of lap $G$ from an ectopic locus caused an $\sim 65 \%$ decrease in peak biofilm formation (SI Appendix, Fig. $\mathrm{S} 3 A$ ) suggesting that enhanced LapG-mediated cleavage of adhesins prematurely released cells from the biofilm. Thus, the conserved Lap pathway, which responds to changes in c-di-GMP level, facilitates biofilm dispersal in $V$. cholerae.

Regarding the RbmB polysaccharide lyase, the $\Delta r b m B$ strain formed biofilms to roughly the same peak biomass as WT; however, it exhibited a 2-h delay in dispersal onset, and most of its biomass $(\sim 70 \%)$ remained at $16 \mathrm{~h}$ (Fig. $3 E$ and Table 1$)$. The level of vpsL-lux expression in the $\Delta r b m B$ mutant was similar to that in the WT, showing that the RbmB dispersal function does not concern production of VPS (Fig. $3 F$ ). Complementation with inducible $r b m B$ expressed from an ectopic locus in the $\Delta r b m B$ strain caused an $\sim 40 \%$ reduction in peak biofilm formation, confirming that $\mathrm{RbmB}$ negatively regulates biofilm formation. However, the complemented strain retained a modest biofilm dispersal defect, suggesting that the timing or level of $r b m B$ expression is critical for WT biofilm disassembly (SI Appendix, Fig. $\mathrm{S} 3 B)$.
To verify that the $\Delta r b m B$ dispersal defect stems from the lack of $v p s$ degradation, we grew $\Delta r b m B$ biofilms for $16 \mathrm{~h}$ (i.e., postWT biofilm dispersal completion), and subsequently fixed and stained the nondispersed biofilms with wheat germ agglutinin conjugated to Texas Red (WGA-txRed), which binds to $N$-acetylglucosamine sugars in the VPS matrix (30). We used the $\Delta l a p G$ mutant as our control, since its biofilm dispersal phenotype should not involve changes in VPS. On average, the $\Delta r b m B$ mutant exhibited approximately sixfold more WGA-txRed signal than the $\Delta$ lapG mutant (Fig. $3 G$ ).

Collectively, our results show that the nondispersed $\Delta l a p G$ biofilms contain little VPS, consistent with possession of functional $\mathrm{RbmB}$, while nondispersed $\Delta r b m B$ biofilms contain excess VPS due to the lack of RbmB-mediated polysaccharide digestion. Thus, we suggest that RbmB-directed VPS disassembly is critical for proper biofilm disassembly (Fig. 3H).

Our results indicate that $\mathrm{LapG}$ and $\mathrm{RbmB}$ function in different pathways to drive biofilm disassembly. To examine their combined effects, we constructed the $\Delta$ lap $G \quad \Delta r b m B$ double mutant and measured its biofilm lifecycle (Fig. 3I). The $\Delta l a p G$ $\Delta r b m B$ double mutant mimicked the single $\Delta r b m B$ mutant (Fig. $3 E$ ) in its biofilm dispersal defect. Thus, the $\Delta l a p G$ and $\Delta r b m B$ defects are not additive. Presumably, the severe dispersal defect displayed by the $\Delta r b m B$ single mutant, which cannot digest matrix polysaccharides, is not made more extreme by additional impairment of matrix protein degradation, suggesting that cells are already maximally trapped by the undigested polysaccharides. It is also possible that an additional factor not identified in the screen exists that is responsible for enabling the $\Delta$ lapG $\Delta r b m B$ double mutant to exhibit some biofilm dispersal ( $\sim 67 \%$ of peak biomass remained at $16 \mathrm{~h}$ ). Alternatively, the subpopulation of cells that successfully dispersed from $\Delta l a p G$

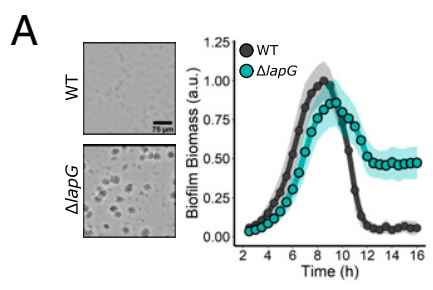

D

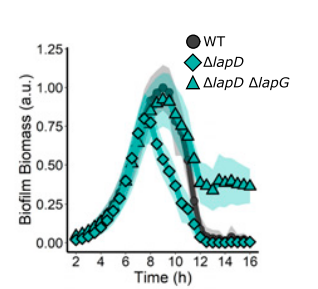

E
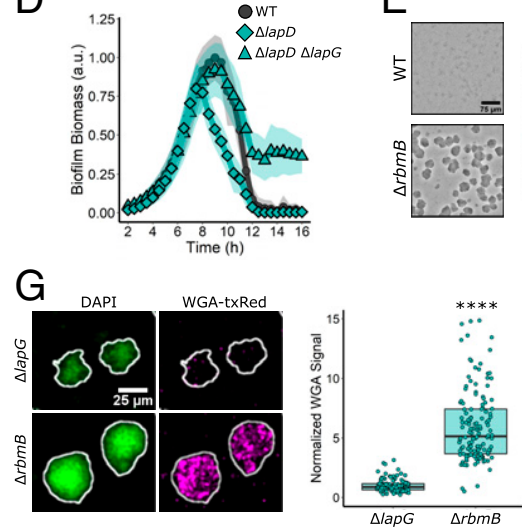
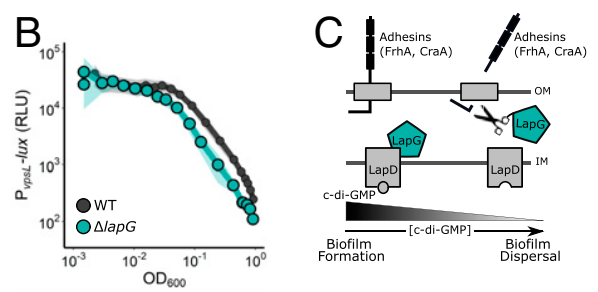

F
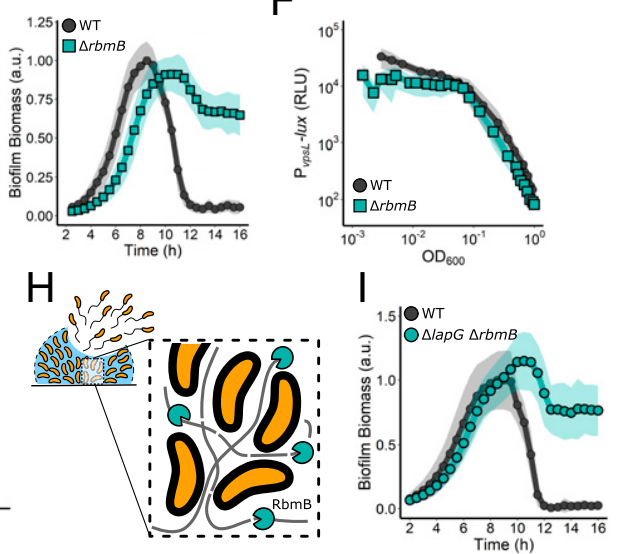

Fig. 3. Matrix-digesting enzymes mediate $V$. cholerae biofilm dispersal. $(A)$ Representative $16 \mathrm{~h}$ images and quantitation of biofilm biomass over time measured by time-lapse microscopy for WT $V$. cholerae and the $\Delta / a p G$ mutant. $(B)$ The corresponding $P_{v p s L}-l u x$ output for strains and growth conditions in $A$ over the growth curve. (C) Schematic representing the LapG mechanism. (D) As in $A$ for the WT, the $\Delta / a p D$ single mutant, and the $\Delta / a p D \Delta / a p G$ double mutant. $(E)$ As in $A$ for the WT and the $\Delta r b m B$ mutant. $(F)$ As in $B$ for the WT and the $\Delta r b m B$ mutant. (G) Representative images and quantitation of WGAtxRed signal in $\Delta$ lapG and $\Delta r b m B$ biofilms at $16 \mathrm{~h}$ postinoculation. To account for differences in biomass, the WGA-txRed signal was divided by the DAPI signal in each biofilm. Values were normalized to the mean signal for the $\Delta$ lapG strain. More than 100 individual biofilms were quantified for each strain. An unpaired $t$ test was performed for statistical analysis. $* * * * P<0.0001$. $(H)$ Proposed model for the role of RbmB in biofilm dispersal. Gray lines represent the polysaccharide matrix. (I) As in $A$ for the WT and the $\Delta$ lapG $\Delta r b m B$ double mutant. In all cases, $n=3$ biological replicates and $n=3$ technical replicates, \pm SD (shaded). For vpsL-lux measurements, $n=3$ biological replicates, \pm SD (shaded). 
$\Delta r b m B$ double-mutant biofilms could be peripherally located cells that are only loosely associated with the biofilm community.

Extracellular DNA (eDNA) is a component of the $V$. cholerae biofilm matrix, and two DNases secreted by $V$. cholerae, Dns and $\mathrm{Xds}$, digest eDNA (31). Although we did not identify $d n s$ or $x d s$ in our screen, we nonetheless investigated whether they contributed to biofilm dispersal. Neither the $\Delta d n s$ and the $\Delta x d s$ single mutants nor the $\Delta d n s \Delta x d s$ double mutant displayed a biofilm dispersal defect in our assay (SI Appendix, Fig. S3C), suggesting that eDNA digestion is not required for dispersal. In a similar vein, we did not identify genes encoding the eight $V$. cholerae extracellular proteases that could degrade matrix proteins. Consistent with this finding, none of the phenotypes of mutants deleted for each extracellular protease gene exhibited a dispersal defect, and thus, no single extracellular protease is required for biofilm dispersal (SI Appendix, Fig. S3D). It remains possible that proteases contribute to biofilm dispersal by functioning redundantly. Taken together, our results indicate that two enzymes, LapG and RbmB, are the primary matrix degrading components that enable biofilm dispersal.

Reorientations in Swimming Direction Are Required for Biofilm Dispersal. The final category of genes identified in our screen are involved in cell motility. As noted above, nonmotile mutants were excluded from our analysis because they are known to be impaired in surface attachment. Nonetheless, we identified a mutant containing a transposon insertion in cheY3 as defective for biofilm dispersal. cheY3 is one of the five $V$. cholerae che $Y$ genes specifying chemotaxis RR proteins (32). Notably, cheY3 is the only $V$. cholerae che $Y$ homolog required for chemotaxis (32). The $\Delta$ cheY3 mutant exhibited similar peak biofilm timing and biomass as WT $V$. cholerae; however, $\sim 21 \%$ of the biomass remained at $16 \mathrm{~h}$ (Fig. $4 A$ and Table 1 ). Complementation via introduction of cheY3 at an ectopic locus restored biofilm dispersal in the mutant (SI Appendix, Fig. S4A). Expression of vpsLlux in the $\Delta$ cheY3 mutant was identical to that in the WT, indicating that the dispersal phenotype was not due to elevated matrix production (Fig. $4 B$ ).

The $V$. cholerae default motor rotation direction is counterclockwise (CCW), which fosters smooth, straight swimming (33). Transition to clockwise $(\mathrm{CW})$ motor rotation causes reorientations in swimming direction (33). Phospho-CheY3 binds to the flagellar motor switch complex to mediate the change from $\mathrm{CCW}$ to $\mathrm{CW}$ rotation. Thus, the $\Delta$ cheY3 mutant is nonchemotactic, and the cells are locked in the CCW straight swimming mode (Fig. $4 C$ ). We reasoned that the $\Delta$ cheY3 mutant dispersal defect could stem from an inability to chemotact or from an inability to reorient swimming direction. To distinguish between these possibilities, we examined biofilm dispersal in a $V$. cholerae mutant carrying a cheY3 allele, cheY3 ${ }^{D 16 K, Y 109 W}$ (hereinafter cheY3*), that locks the motor into $\mathrm{CW}$ rotation and so also disrupts chemotaxis. cheY $3^{*}$ cells undergo frequent reorientations and are unable to swim in smooth, straight runs (Fig. 4C) $(32,34)$. The cheY3* strain has WT biofilm dispersal capability (Fig. $4 A$ ). Thus, being chemotactic is not required for $V$. cholerae to exit biofilms.

We reasoned that analysis of the unique motility characteristics of our strains could reveal the underlying causes of the $\Delta c h e Y 3$ biofilm dispersal defect, and thus we measured the turning frequencies and swimming velocities of the WT, $\Delta$ cheY3, and $c h e Y 3^{*} V$. cholerae strains. Consistent with previous reports, these three strains exhibited notable differences; on average, the WT turned once every $3 \mathrm{~s}$, the $\Delta c h e Y 3$ mutant turned less than once every $40 \mathrm{~s}$, and the cheY3* strain turned once every $0.5 \mathrm{~s}$ (Fig. $4 C$ and $D)(32,34)$. The cheY3* strain displayed slightly lower average swimming velocity than the WT and $\Delta$ cheY3 strains due to its high turning frequency, as turning necessarily involves a decrease in velocity (Fig. $4 E$ ) (35). Together, these
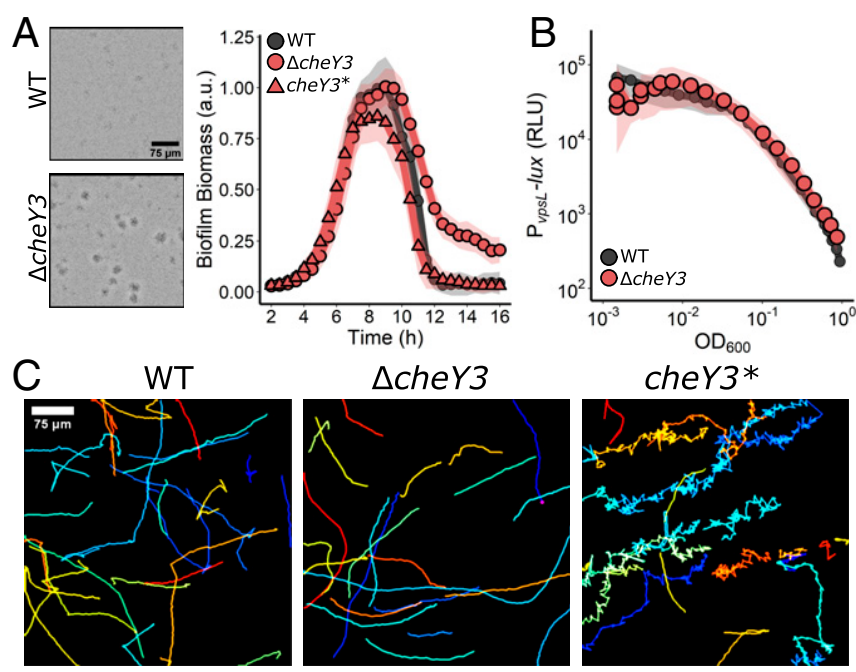

cheY3*
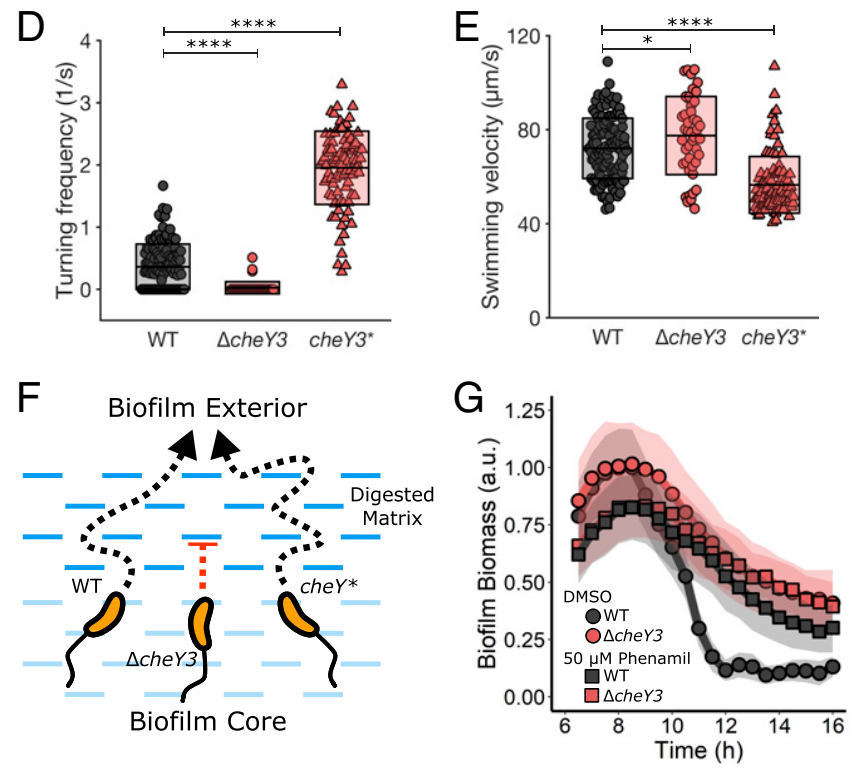

Fig. 4. Reorientations in swimming direction are required for $V$. cholerae biofilm dispersal. $(A)$ Representative $16 \mathrm{~h}$ images and quantitation of biofilm biomass over time measured by time-lapse microscopy for WT $V$. cholerae, the $\Delta$ cheY3 mutant, and the cheY3 ${ }^{D 16 K, Y 109 W}$ (cheY3*) mutant. (B) The corresponding $P_{\text {vpsL }}-/ u x$ output for WT and the $\Delta$ cheY3 strain over the growth curve. $(C)$ Representative, randomly colored, single-cell locomotion trajectories for the strains in $A$. (D) Turning frequencies of the strains in $A$. $(E)$ Measured swimming velocities of the strains in $A$. ( $F$ ) Proposed model for the role of motility and reorientation in biofilm dispersal. $(G)$ Quantitation of biofilm biomass over time for WT and the $\Delta$ cheY3 mutant following treatment with DMSO or the motility inhibitor phenamil supplied at $5 \mathrm{~h}$ postinoculation. For biofilm biomass assays, $n=3$ biological replicates and $n=3$ technical replicates, \pm SD (shaded). For vpsL-lux measurements, $n=3$ biological replicates, \pm SD (shaded). For motility measurements, 45 to 125 individual cells of each strain were tracked. In $D$ and $E$, unpaired $t$ tests were performed for statistical analysis. ${ }^{\star} P<0.05 ; * * P<0.01 ; * * * P<0.001$; $\star * * * P<0.0001 ;$ n.s., $P>0.05$.

results suggest that the low turning frequency of the $\Delta$ cheY3 mutant is responsible for the biofilm dispersal defect. We propose that if cells do not frequently change their direction of motion, they become trapped by the biofilm matrix mesh, which compromises their ability to escape (Fig. $4 F$ ). Indeed, in other bacteria, straight-swimming mutants are deficient in traversing fluid-filled porous media compared with WT organisms that can reorient (36). 
These results indicate that chemotaxis itself is not required for biofilm dispersal, but the chemotaxis machinery facilitates random reorientation events that allow $V$. cholerae cells to navigate a porous biofilm matrix. The same nonchemotactic mutants used here exhibit stark differences in competition experiments in animal models of cholera infection, demonstrating that their differences in motility, and possibly also in biofilm dispersal capability, are pertinent to colonization (34).

Finally, we determined whether the ability to locomote was required for biofilm dispersal or, by contrast, if nonmotile cells could escape the digested matrix via Brownian motion. As mentioned above, we could not simply study dispersal of nonflagellated and nonmotile mutants because of their confounding surface attachment defects and feedback on biofilm regulatory components $(37,38)$. To circumvent this problem, we used phenamil, an inhibitor of the $\mathrm{Na}^{+}$-driven $V$. cholerae flagellar motor, which dramatically reduced planktonic cell motility, as expected (SI Appendix, Fig. S4B) (39). To assess the role of swimming motility in biofilm dispersal, we first allowed WT $V$. cholerae cells to undergo biofilm formation for $5 \mathrm{~h}$, at which point we perfused DMSO or phenamil into the incubation chamber (Fig. 4G). Following phenamil treatment, the WT strain displayed a dispersal defect nearly identical to that of the $\Delta c h e Y 3$ mutant. In addition, phenamil treatment of the $\Delta$ cheY3 mutant did not further impair its biofilm dispersal. Together, these results demonstrate that swimming motility is crucial for $V$. cholerae biofilm dispersal, and that an inability to reorient is as detrimental to dispersal as a complete lack of flagellar motility.

\section{Discussion}

Previous studies provided initial clues concerning the regulation of $V$. cholerae biofilm dispersal: quorum sensing, the process of cell-to-cell communication that bacteria use to orchestrate collective behaviors, bile salts, and starvation signals promote dispersal $(18,19,40,41)$. However, other components, and specifically those that enable the cells to exit the biofilms, remained largely unknown. In this study, we developed a highcontent imaging screen that allowed us to identify components required for $V$. cholerae biofilm dispersal. We categorized the identified components into three classes: signal transduction, matrix disassembly, and cell motility. We propose that the three functional categories represent the chronological steps required for the disassembly of a biofilm. First, the stimuli that activate dispersal must accumulate. Subsequently, the gene expression pattern established by detection of these stimuli must repress biofilm matrix production and activate production of enzymes required to digest the biofilm matrix. Finally, cells must escape through the partially digested, porous matrix, which requires changes in the direction of movement.

Here we show that the DbfS-DbfR two-component system controls matrix production. While not yet tested explicitly, it is possible that DbfS-DbfR also orchestrates the initiation of matrix digestion and the launch of motility. Together, these steps ensure that when environmental conditions are appropriate, $V$. cholerae cells can exit the sessile lifestyle and disseminate to new terrain that is ripe for biofilm formation or, alternatively during disease, to a new host. One can now imagine targeting the functions identified in this work for small-molecule disruption of the $V$. cholerae biofilm lifecycle, possibly guiding the development of treatments to reduce the duration of $V$. cholerae infection or to prevent transmission.

\section{Materials and Methods}

Bacterial Strains and Reagents. The $V$. cholerae parent strain used in this study was WT 01 El Tor biotype C6706str2. Antibiotics were used at the following concentrations: polymyxin B, $50 \mu \mathrm{g} / \mathrm{mL}$; kanamycin, $50 \mu \mathrm{g} / \mathrm{mL}$; spectinomycin, $200 \mu \mathrm{g} / \mathrm{mL}$; and chloramphenicol, $1 \mu \mathrm{g} / \mathrm{mL}$ Strains were propagated in lysogeny broth (LB) supplemented with $1.5 \%$ agar or in liquid
LB with shaking at $30{ }^{\circ} \mathrm{C}$. All strains used in this work are reported in $\mathrm{SI}$ Appendix, Table S1. Unless stated otherwise, exogenous compounds were added from the onset of biofilm initiation. The antimicrobial peptide C18G (VWR) was added at $5 \mu \mathrm{g} / \mathrm{mL}$. Phenamil (Sigma-Aldrich) was prepared in DMSO and added at $5 \mathrm{~h}$ post-biofilm inoculation to a final concentration of $50 \mu \mathrm{M}$. L-arabinose (Sigma-Aldrich) was prepared in water and added at $0.2 \%$.

DNA Manipulation and Strain Construction. To produce linear DNA fragments for natural transformations, splicing overlap extension PCR was performed using iProof polymerase (Bio-Rad) to combine DNA pieces. Primers and gene fragments used in this study are reported in SI Appendix, Table S2. In all cases, $\sim 3 \mathrm{~kb}$ of upstream and downstream flanking regions of homology were generated by PCR from $V$. cholerae genomic DNA and were included to ensure high chromosomal integration frequency. DNA fragments that were not native to $V$. cholerae were synthesized as g-blocks (IDT).

All $V$. cholerae strains generated in this work were constructed by replacing genomic DNA with DNA introduced by natural transformation as described previously $(18,42)$. The neutral vc1807 locus was used as the site of introduction of the gene encoding the antibiotic resistance cassette in the natural cotransformation procedure. The vc1807 locus also served as the site for the introduction of genes under study in chromosomal ectopic expression analyses (42). PCR and Sanger sequencing were used to verify correct integration events. Genomic DNA from recombinant strains was used for future cotransformations and as templates for PCR to generate DNA fragments when necessary. Deletions were constructed in frame and eliminated the entire coding sequences. The exceptions were $m b a A, d b f S$, and $d b f R$, each of which overlaps with another gene in their operons. In these cases, portions of the genes were deleted ensuring that adjacent genes were not perturbed. For $\operatorname{tag} A$, the first 103 base pairs, including the nucleotides specifying the start codon, were deleted. All strains constructed in this study were verified by sequencing at GENEWIZ.

Microscopy and Mutant Screening. The biofilm lifecycle was measured using time-lapse microscopy as described previously (18). All plots were generated using ggplot2 in R. To generate the library of $V$. cholerae insertion mutants for the dispersal screen, the WT parent strain was mutagenized with Tn5 as described previously (43). Mutants were selected by growth overnight on LB plates containing polymyxin B and kanamycin. The next day, mutant colonies were arrayed into 96-well plates containing $200 \mu \mathrm{L}$ of LB medium supplemented with polymyxin B and kanamycin using an automated colony-picking robot (Molecular Devices). The arrayed cultures were grown in a plate-shaking incubator at $30{ }^{\circ} \mathrm{C}$ covered with Breathe-Easy membranes (Sigma-Aldrich) to minimize evaporation. After $16 \mathrm{~h}$ of growth, the arrayed cultures were diluted 1:200,000 into 96-well plates containing M9 medium supplemented with glucose and casamino acids. Diluted cultures were incubated statically at $30^{\circ} \mathrm{C}$ for $8 \mathrm{~h}$ (to achieve peak biofilm biomass), at which point images of each well were captured on a Nikon Ti-E inverted microscope using transmitted-light bright-field illumination, a 10x Plan Fluor (NA 0.3) objective lens, and an Andor iXon 897 EMCCD camera. Automated image acquisition was performed using NISElements software v5.11.02 and the NIS-Elements Jobs Module to acquire images at four positions within each well to account for heterogeneity within samples. The focal plane between wells was maintained using the Nikon Perfect Focus system.

After microscopy at the $8 \mathrm{~h}$ timepoint, 96-well plates were returned to the incubator. To assess biofilm dispersal, a second set of images of the same samples was acquired at $13 \mathrm{~h}$ postinoculation. Mutants that displayed biofilm growth at the $8 \mathrm{~h}$ timepoint but failed to disperse by the $13 \mathrm{~h}$ timepoint were subcultured, grown overnight, and subsequently reimaged using the timelapse approach described above to assess their biofilm lifecycles in real time. Mutants that exhibited biofilm dispersal defects after this reassessment step were analyzed for the locations of transposon insertions using arbitrary PCR (44)

vpsL-Iux Transcription Assay. Three colonies of each strain to be analyzed were individually grown overnight in $200 \mu \mathrm{L}$ of LB with shaking at $30{ }^{\circ} \mathrm{C}$ in a 96-well plate covered with a Breathe-Easy membrane. The next morning, the cultures were diluted 1:5,000 into fresh M9 medium supplemented with glucose and casamino acids. The plates were placed in a BioTek Synergy $\mathrm{Neo} 2 \mathrm{Multi}-M o d e$ reader under static growth conditions at $30^{\circ} \mathrm{C} . \mathrm{OD}_{600}$ and bioluminescence from vpsL-lux were measured simultaneously at 15-min time intervals. The results were exported to $R$, and light values were divided by $\mathrm{OD}_{600}$ to produce relative light units (RLUs). Results from replicates were averaged and plotted using ggplot 2 in $R$. 
VPS Quantitation. To assess VPS levels in nondispersed biofilms using WGAtxRED, biofilms were grown for $16 \mathrm{~h}$ and then washed three times with $1 \times$ PBS and fixed for 10 min with $3.7 \%$ formaldehyde in $1 \times$ PBS. After fixation, samples were washed five times with $1 \times$ PBS and subsequently incubated with a solution containing $1 \mu \mathrm{g} / \mathrm{mL}$ WGA-txRED (Thermo Fisher Scientific), $1 \mu \mathrm{g} / \mathrm{mL} \mathrm{DAPI}$, and $1 \% \mathrm{BSA}$ in $1 \times \mathrm{PBS}$ for $1 \mathrm{~h}$ with shaking at $30^{\circ} \mathrm{C}$ in the dark. After incubation, samples were washed five more times with 1× PBS before imaging. Confocal microscopy was performed with a Leica DMI8 SP-8 point scanning confocal microscope with the pinhole set to 1.0 airy unit. The light source for DAPI was a 405-nm laser, and the light source used to excite WGA-txRED was a tunable white-light laser (Leica model WLL2; excitation window, 470 to $670 \mathrm{~nm}$ ) set to $595 \mathrm{~nm}$. Biofilms were imaged using a 10× air objective (Leica, HC PL FLUOTAR; NA 0.30). Sequential frame scanning was performed to minimize spectral bleed-through in images. Emitted light was detected using GaAsP spectral detectors (Leica HyD SP), and timed gate detection was used to minimize the background signal. Image analyses were performed with Fiji version $1.52 \mathrm{p}$. Biofilms were segmented in the DAPI channel using an intensity threshold, and the intensities of the channels were measured. The same threshold was applied to all images. The WGAtxRED signal was divided by the DAPI signal to achieve the normalized WGA signal.

Motility Assay. To prevent biofilm formation during measurements of swimming velocities and turning frequencies for the WT, $\Delta c h e Y 3$, and cheY3* strains, vpsL was deleted. Each strain was grown for $16 \mathrm{~h}$ in LB medium. The next day, cells were diluted to $\mathrm{OD}_{600}=0.001$ in $\mathrm{M} 9$ medium supplemented with glucose and casamino acids. Subsequently, diluted cultures were dispensed in 200- $\mu \mathrm{L}$ aliquots into glass coverslip-bottomed 96-well plates (MatTek). After $1 \mathrm{~h}$, during which time cells were allowed to adhere to the coverslips, wells were washed eight times with fresh medium to remove unattached cells. The plates were incubated at $25^{\circ} \mathrm{C}$ for $3 \mathrm{~h}$, and imaging was performed using the brightfield setup described above for the biofilm dispersal screen. In this case, the frame interval was $50 \mathrm{~ms}$, and imaging was conducted at a distance of $\sim 100 \mu \mathrm{m}$ into the sample. Images were smoothed, background-corrected, and imported into the TrackMate $(\mathrm{v}$ 5.2.0) plugin in Fiji. Cells were detected with a Laplacian of Gaussian detector and subsequently tracked using the simple linear assignment problem approach. To exclude nonmotile cells from our analyses in Fig. $4 C-E$, objects with velocity $<40 \mu \mathrm{m} / \mathrm{s}$ were eliminated. Analyses and plotting of swimming velocities and turning frequencies were performed in MATLAB (MathWorks).

1. C. Matz et al., Biofilm formation and phenotypic variation enhance predation-driven persistence of Vibrio cholerae. Proc. Natl. Acad. Sci. U.S.A. 102, 16819-16824 (2005).

2. J. Yan, B. L. Bassler, Surviving as a community: Antibiotic tolerance and persistence in bacterial biofilms. Cell Host Microbe 26, 15-21 (2019).

3. T. Shaw, M. Winston, C. J. Rupp, I. Klapper, P. Stoodley, Commonality of elastic relaxation times in biofilms. Phys. Rev. Lett. 93, 098102 (2004).

4. J. Walter, R. A. Britton, S. Roos, Host-microbial symbiosis in the vertebrate gastrointestinal tract and the Lactobacillus reuteri paradigm. Proc. Natl. Acad. Sci. U.S.A. 108 (suppl. 1), 4645-4652 (2011).

5. E. Roilides, M. Simitsopoulou, A. Katragkou, T. J. Walsh, How biofilms evade host defenses. Microbiol. Spectr. 3, (2015).

6. T. F. Mah, G. A. O'Toole, Mechanisms of biofilm resistance to antimicrobial agents. Trends Microbiol. 9, 34-39 (2001)

7. P. L. Phillips, G. S. Schultz, Molecular mechanisms of biofilm infection: Biofilm virulence factors. Adv. Wound Care (New Rochelle) 1, 109-114 (2012).

8. C. Guilhen, C. Forestier, D. Balestrino, Biofilm dispersal: Multiple elaborate strategies for dissemination of bacteria with unique properties. Mol. Microbiol. 105, 188-210 (2017).

9. L. Hobley, C. Harkins, C. E. MacPhee, N. R. Stanley-Wall, Giving structure to the biofilm matrix: An overview of individual strategies and emerging common themes. FEMS Microbiol. Rev. 39, 649-669 (2015).

10. J. G. Conner, J. K. Teschler, C. J. Jones, F. H. Yildiz, Staying alive: Vibrio cholerae's cycle of environmental survival, transmission, and dissemination. Microbiol. Spectr. 4 (2016).

11. S. Almagro-Moreno, K. Pruss, R. K. Taylor, Intestinal colonization dynamics of Vibrio cholerae. PLoS Pathog. 11, e1004787 (2015)

12. A. J. Silva, J. A. Benitez, Vibrio cholerae biofilms and cholera pathogenesis. PLoS Negl. Trop. Dis. 10, e0004330 (2016).

13. A. L. Gallego-Hernandez et al., Upregulation of virulence genes promotes Vibrio cholerae biofilm hyperinfectivity. Proc. Natl. Acad. Sci. U.S.A. 117, 11010-11017 (2020).

14. R. Tamayo, B. Patimalla, A. Camilli, Growth in a biofilm induces a hyperinfectious phenotype in Vibrio cholerae. Infect. Immun. 78, 3560-3569 (2010)

15. M. Valentini, A. Filloux, Biofilms and cyclic di-GMP (c-di-GMP) signaling: Lessons from Pseudomonas aeruginosa and other bacteria. J. Biol. Chem. 291, 12547-12555 (2016)
Local curvatures for single-cell locomotion trajectories were calculated as described previously (45). A curvature $<0.3 \mu \mathrm{m}^{-1}$ was used to identify the turning events. Mean squared displacement was calculated as described previously (46).

Phos-Tag Gel Analysis. To monitor DbfR and phospho-DbfR via sodium dodecyl sulfate polyacrylamide gel electrophoresis (SDS/PAGE), the endogenous $d f b R$ gene was replaced with $d b f R-S N A P$ in the $\triangle d b f S$ strain, and $P_{B A D}-d b f S$ was introduced at the ectopic locus, vc1807. To assess DbfR-SNAP phosphorylation in the absence and presence of DbfS, overnight cultures of the strain were diluted $1: 1,000$ and subsequently grown for $4 \mathrm{~h}$ at $30^{\circ} \mathrm{C}$ with shaking to an $\mathrm{OD}_{600}$ of $\sim 0.6$. To each culture, $1 \mu \mathrm{M}$ SNAP-Cell TMR Star (New England BioLabs) was added to label the SNAP tag, and the culture was subsequently divided into two tubes. Subsequently, $0.2 \% \mathrm{D}$-fucose was added to one tube, and $0.2 \%$ L-arabinose was added to the other tube, to repress and induce DbfS production, respectively. The cultures were returned to $30{ }^{\circ} \mathrm{C}$ with shaking. After $1 \mathrm{~h}$, the cells were collected by centrifugation for $1 \mathrm{~min}$ at 13,000 rpm. Lysis and solubilization were carried out as rapidly as possible. In brief, cells were chemically lysed by resuspension to $\mathrm{OD}_{600}=1.0$ in $40 \mu \mathrm{L}$ of Bug Buster (Novagen) for $5 \mathrm{~min}$ at $25^{\circ} \mathrm{C}$ with intermittent vortex. The cell lysate was solubilized at $25^{\circ} \mathrm{C}$ in $1.5 \times$ SDS/PAGE buffer for $5 \mathrm{~min}$ also with intermittent vortex. Samples were immediately loaded onto a cold 7.5\% SuperSep Phos-tag $(50 \mu \mathrm{M})$ gel (FUJIFILM Wako Pure Chemical; 198-17981). Electrophoresis was carried out at $100 \mathrm{~V}$ at $4{ }^{\circ} \mathrm{C}$ until the loading buffer exited the gel. Gel images were captured on an ImageQuant LAS 4000 imager (GE Healthcare) using a Cy3 filter set.

Data Availability. All study data are included in the main text and supporting information.

ACKNOWLEDGMENTS. We thank members of the Bassler group and Ned Wingreen for thoughtful discussions. We particularly thank Matthew Jemielita for help with the secreted protease mutants used in this study This work was supported by the HHMI, NIH (Grant 5R37GM065859), NSF (Grant MCB-1713731), and a Max Planck-Alexander von Humboldt research award (to B.L.B.). A.A.B. is an HHMI Fellow of the Damon Runyon Cancer Research Foundation (DRG-2302-17). The content is solely the responsibility of the authors and does not necessarily represent the official views of the $\mathrm{NIH}$. The funders had no role in study design, data collection and analysis, decision to publish, or manuscript preparation.

16. J. G. Conner, D. Zamorano-Sánchez, J. H. Park, H. Sondermann, F. H. Yildiz, The ins and outs of cyclic di-GMP signaling in Vibrio cholerae. Curr. Opin. Microbiol. 36, 20-29 (2017).

17. J. C. N. Fong, K. A. Syed, K. E. Klose, F. H. Yildiz, Role of Vibrio polysaccharide (vps) genes in VPS production, biofilm formation and Vibrio cholerae pathogenesis. Microbiology 156, 2757-2769 (2010).

18. A. A. Bridges, B. L. Bassler, The intragenus and interspecies quorum-sensing autoinducers exert distinct control over Vibrio cholerae biofilm formation and dispersal. PLoS Biol. 17, e3000429 (2019).

19. P. K. Singh et al., Vibrio cholerae combines individual and collective sensing to trigger biofilm dispersal. Curr. Biol. 27, 3359-3366.e7 (2017).

20. D. S. Merrell, D. L. Hava, A. Camilli, Identification of novel factors involved in colonization and acid tolerance of Vibrio cholerae. Mol. Microbiol. 43, 1471-1491 (2002).

21. R. Gao, A. M. Stock, Biological insights from structures of two-component proteins. Annu. Rev. Microbiol. 63, 133-154 (2009).

22. R. Gao, S. Bouillet, A. M. Stock, Structural basis of response regulator function. Annu. Rev. Microbiol. 73, 175-197 (2019).

23. C. M. Waters, W. Lu, J. D. Rabinowitz, B. L. Bassler, Quorum sensing controls biofilm formation in Vibrio cholerae through modulation of cyclic di-GMP levels and repression of vpsT. J. Bacteriol. 190, 2527-2536 (2008).

24. C. Yeats, N. D. Rawlings, A. Bateman, The PepSY domain: A regulator of peptidase activity in the microbial environment? Trends Biochem. Sci. 29, 169-172 (2004).

25. C. D. Boyd, D. Chatterjee, H. Sondermann, G. A. O'Toole, G. Lap, LapG, required for modulating biofilm formation by Pseudomonas fluorescens PfO-1, is a calciumdependent protease. J. Bacteriol. 194, 4406-4414 (2012).

26. M. Gjermansen, P. Ragas, C. Sternberg, S. Molin, T. Tolker-Nielsen, Characterization of starvation-induced dispersion in Pseudomonas putida biofilms. Environ. Microbiol. 7, 894-906 (2005)

27. J. C. N. Fong, F. H. Yildiz, The $r b m B C D E F$ gene cluster modulates development of rugose colony morphology and biofilm formation in Vibrio cholerae. J. Bacteriol. 189, 2319-2330 (2007).

28. G. Kitts et al., A conserved regulatory circuit controls large adhesins in Vibrio cholerae. MBio 10, e02822-19 (2019)

29. A. J. Collins, T. J. Smith, H. Sondermann, G. A. O'Toole, From input to output: The lap/ c-di-GMP biofilm regulatory circuit. Annu. Rev. Microbiol. 74, 607-631 (2020).

30. V. Berk et al., Molecular architecture and assembly principles of Vibrio cholerae biofilms. Science 337, 236-239 (2012) 
31. A. Seper et al., Extracellular nucleases and extracellular DNA play important roles in Vibrio cholerae biofilm formation. Mol. Microbiol. 82, 1015-1037 (2011).

32. A. Hyakutake et al., Only one of the five CheY homologs in Vibrio cholerae directly switches flagellar rotation. J. Bacteriol. 187, 8403-8410 (2005).

33. S. M. Butler, A. Camilli, Going against the grain: Chemotaxis and infection in Vibrio cholerae. Nat. Rev. Microbiol. 3, 611-620 (2005).

34. S. M. Butler, A. Camilli, Both chemotaxis and net motility greatly influence the infectivity of Vibrio cholerae. Proc. Natl. Acad. Sci. U.S.A. 101, 5018-5023 (2004).

35. K. Son, J. S. Guasto, R. Stocker, Bacteria can exploit a flagellar buckling instability to change direction. Nat. Phys. 9, 494-498 (2013).

36. N. A. Licata, B. Mohari, C. Fuqua, S. Setayeshgar, Diffusion of bacterial cells in porous media. Biophys. J. 110, 247-257 (2016).

37. A. S. Utada et al., Vibrio cholerae use pili and flagella synergistically to effect motility switching and conditional surface attachment. Nat. Commun. 5, 4913 (2014).

38. D. C. Wu et al., Reciprocal c-di-GMP signaling: Incomplete flagellum biogenesis triggers c-di-GMP signaling pathways that promote biofilm formation. PLoS Genet. 16 e1008703 (2020).
39. S. Kojima, K. Yamamoto, I. Kawagishi, M. Homma, The polar flagellar motor of Vibrio cholerae is driven by an $\mathrm{Na}^{+}$motive force. J. Bacteriol. 181, 1927-1930 (1999).

40. Z. Liu, F. R. Stirling, J. Zhu, Temporal quorum-sensing induction regulates Vibrio cholerae biofilm architecture. Infect. Immun. 75, 122-126 (2007).

41. A. J. Hay, J. Zhu, Host intestinal signal-promoted biofilm dispersal induces Vibrio cholerae colonization. Infect. Immun. 83, 317-323 (2015)

42. A. B. Dalia, E. McDonough, A. Camilli, Multiplex genome editing by natural transformation. Proc. Natl. Acad. Sci. U.S.A. 111, 8937-8942 (2014).

43. M. B. Miller, K. Skorupski, D. H. Lenz, R. K. Taylor, B. L. Bassler, Parallel quorum sensing systems converge to regulate virulence in Vibrio cholerae. Cell 110, 303-314 (2002)

44. J. T. Saavedra, J. A. Schwartzman, M. S. Gilmore, Mapping transposon insertions in bacterial genomes by arbitrarily primed PCR. Curr. Protoc. Mol. Biol. 118, 15.15.1-15.15.15 (2017)

45. J. Yan et al., Mechanical instability and interfacial energy drive biofilm morphogenesis. eLife 8, e43920 (2019).

46. B. Qin et al., Cell position fates and collective fountain flow in bacterial biofilms revealed by light-sheet microscopy. Science 369, 71-77 (2020). 